Mon. Not. R. Astron. Soc. 000, 1-11 (2016) Printed 20 September $2018 \quad$ (MN LTEX style file v2.2)

\title{
Fragmentation inside atomic cooling haloes exposed to Lyman-Werner radiation
}

\author{
John A. Regan` \& Turlough P. Downes \\ Centre for Astrophysics \& Relativity, School of Mathematical Sciences, Dublin City University, Glasnevin, Ireland
}

20 September 2018

\begin{abstract}
Supermassive stars born in pristine environments in the early Universe hold the promise of being the seeds for the supermassive black holes observed as high redshift quasars shortly after the epoch of reionisation. $\mathrm{H}_{2}$ suppression is thought to be crucial in order to negate normal Population III star formation and allow high accretion rates to drive the formation of supermassive stars. Only in the cases where vigorous fragmentation is avoided will a monolithic collapse be successful giving rise to a single massive central object. We investigate the number of fragmentation sites formed in collapsing atomic cooling haloes subject to various levels of background Lyman-Werner flux. The background Lyman-Werner flux manipulates the chemical properties of the gas in the collapsing halo by destroying $\mathrm{H}_{2}$. We find that only when the collapsing gas cloud shifts from the molecular to the atomic cooling regime is the degree of fragmentation suppressed. In our particular case we find that this occurs above a critical Lyman-Werner background of $\mathbf{J} \sim 10 \mathrm{~J}_{21}$. The important criterion being the transition to the atomic cooling regime rather than the actual value of $\mathrm{J}$, which will vary locally. Once the temperature of the gas exceeds $\mathrm{T} \gtrsim 10^{4} \mathrm{~K}$ and the gas transitions to atomic line cooling, then vigorous fragmentation is strongly suppressed.
\end{abstract}

Key words: Cosmology: theory - large-scale structure - first stars, methods: numerical

\section{INTRODUCTION}

Supermassive black holes (SMBHs) populate the centres of massive galaxies (Kormendy \& Ho 2013) and shine as extremely luminous quasars out to high redshift (Fan et al. 2006; Mortlock et al. 2011; Wu et al. 2015). Their progenitors are unknown, however, with competing theories for whether the progenitors of SMBHs were stellar mass black holes or supermassive stars with initial masses of $\mathrm{M}_{* \text {, init }} \gtrsim 10^{4} \mathrm{M}_{\odot}$ (e.g. Loeb \& Rasio 1994; Madau \& Rees 2001). Stellar mass black holes formed from the remnants of the first stars (PopIII stars) must accrete at the Eddington limit for their entire history to reach the billion mass threshold by a redshift of 7 if they are to be the seeds of the first quasars. This scenario appears exceedingly difficult as the first stellar mass black holes are born "starving" (Whalen et al. 2004; Alvarez et al. 2009; Johnson et al. 2011) in mini haloes which have been disrupted by both the ionising radiation from PopIII stars and the subsequent supernova explosions (Milosavljević et al. 2009; Jeon et al. 2014). As a direct result, investigation of supermassive star (SMS) formation as a viable alternative has been undertaken and appears attractive (Haiman 2006; Begelman et al. 2006; Wise et al.

^ E-mail:john.regan@dcu.ie, Marie Skłdowska-Curie Fellow
2008; Regan \& Haehnelt 2009a,b; Volonteri 2010; Agarwal et al. 2012, 2013, 2014; Latif \& Volonteri 2015; Latif et al. 2016; Regan et al. 2017). The larger initial seed masses of SMSs alleviate the growth requirements somewhat (Tanaka \& Haiman 2009). More fundamentally however, is the fact that the SMS is born in a much larger halo compared to the canonical PopIII case. SMS formation is thought to be possible only in pristine atomic cooling haloes with virial temperatures $\mathrm{T}_{v i r} \gtrsim 10^{4} \mathrm{~K}$ with accretion rates exceeding $\dot{M} \gtrsim 0.1 \mathrm{M}_{\odot} /$ yr (Hosokawa et al. 2013a; Schleicher et al. 2013; Hosokawa et al. 2013b). The larger halo leads to a much deeper potential well relative to the minihalo case, the SMS is also expected to collapse directly into a black hole skipping the supernovae phase and what's more the radiation spectrum expected from a SMS peaks in the infrared rather than the UV as is the case for PopIII stars(Hosokawa et al. 2016). Taken together, SMSs appear to offer far more favourable conditions for being the original seeds for SMBHs.

The conditions for achieving SMS formation are, however, not yet fully understood. It is widely suspected that high mass accretion rates combined with high temperature gas will lead to the formation of a SMS. This can be achieved in a number of ways. LymanWerner (LW) radiation from a nearby galaxy will disrupt $\mathrm{H}_{2}$ abundances removing a coolant, allowing a halo to grow sufficiently 
Table 1. Simulation Parameters

\begin{tabular}{lcclccc}
\hline Sim Name $^{a}$ & $\boldsymbol{J}_{21}^{b}$ & Maximum Resolution $^{c}(\boldsymbol{p c})$ & Collapse Redshift $^{d}$ & Num Fragments $^{e}$ & $\boldsymbol{M}_{\text {core }}^{f}\left(\mathrm{M}_{\odot}\right)$ & $\boldsymbol{M}_{\text {halo }}^{g}\left(\mathrm{M}_{\odot}\right)$ \\
\hline Ctr1_Ref16 & 0.0 & 0.01 & $\mathrm{z}=32.68$ & $1,1,2$ & 2186 & $1.07 \times 10^{6}$ \\
1J21_Ref16 & 1.0 & 0.01 & $\mathrm{z}=30.55$ & $1,3,5$ & 6571 & $3.00 \times 10^{6}$ \\
2J21_Ref16 & 2.0 & 0.01 & $\mathrm{z}=29.91$ & $2,5,6$ & 5101 & $3.72 \times 10^{6}$ \\
4J21_Ref16 & 4.0 & 0.01 & $\mathrm{z}=28.87$ & $5,6,4$ & 9219 & $5.55 \times 10^{6}$ \\
4J21_Ref20 & 4.0 & 0.004 & $\mathrm{z}=28.87$ & $22,-,-$ & 12386 & $5.56 \times 10^{6}$ \\
4J21_Ref24 & 4.0 & 0.001 & $\mathrm{z}=28.87$ &,,--- & 12405 & $5.56 \times 10^{6}$ \\
10J21_Ref16 & 10.0 & 0.01 & $\mathrm{z}=27.15$ & $1,1,4$ & 12917 & $1.21 \times 10^{7}$ \\
50J21_Ref16 & 50.0 & 0.01 & $\mathrm{z}=25.36$ & $2,4,3$ & 15157 & $1.22 \times 10^{7}$ \\
100J21_Ref16 & 100.0 & 0.01 & $\mathrm{z}=24.73$ & $1,1,1$ & 16648 & $1.38 \times 10^{7}$ \\
100J21_Ref20 & 100.0 & 0.004 & $\mathrm{z}=24.72$ & $4,-,-$ & 20169 & $1.38 \times 10^{7}$ \\
100J21_Ref24 & 100.0 & 0.001 & $\mathrm{z}=24.72$ &,,--- & 20359 & $1.38 \times 10^{7}$ \\
\hline
\end{tabular}

Notes: The details of each of the realisations used in this study. (a) The simulation name, (b) The LW intensity in units of $\mathrm{J}_{21}$, (c) the maximum comoving resolution, (d) the collapse redshift (i.e. the redshift at which the first smartstar forms), (e) The number of fragments at 80 kyr, $135 \mathrm{kyr}$ and $650 \mathrm{kyr}$ respectively (f) mass within the central $1 \mathrm{pc}$ just before the first SmartStar forms (i.e. initial mass surrounding the fragmentation site) in solar masses and (g) is the halo virial mass in solar masses. Note that for the higher resolution runs (i.e. those with 20 and 24 levels of refinement) we were not able to follow the evolution for more than 80 kyrs for the 20 levels run and only for a few tens of kyrs for the 24 level runs.

without PopIII formation (Shang et al. 2010; Regan et al. 2014b, 2016). Haloes which accrete unusually rapidly through a succession of mergers provide another pathway(Yoshida et al. 2003) as do streaming velocities(Tanaka \& Li 2014; Schauer et al. 2017) left over from the decoupling of baryons and dark matter after recombination. A combination of one or more of these scenarios is also possible. We focus here on haloes which are exposed to a nearby source of LW radiation as it illustrates the necessary points and is straightforward to implement. The fragmentation of gas inside a collapsing halo could potentially disrupt the formation of a SMS if the gas were to fragment into sufficiently distant clumps to avoid the formation of a single (or small multiple) of very massive stars at the centre of a collapsing atomic cooling halo.

We note that any mechanism (with or without a LW component) which suppresses PopIII star formation and induces the same kind of thermodynamic change to the gas as an external LW field will also likely impact fragmentation in the same way. While the fragmentation of primordial haloes has been thoroughly investigated for the case of PopIII formation(e.g. Bromm et al. 1999; Abel et al. 2000; Clark et al. 2011; Stacy et al. 2016; Safranek-Shrader et al. 2016) it has not been as systematically probed for the case of irradiated haloes (but see Safranek-Shrader et al. (2012), Latif et al. (2014) (L14b), Regan et al. (2014a) and Inayoshi \& Haiman (2014) for some preliminary work). Here we investigate this scenario more systematically, varying the intensity of the radiation and investigating the degree of fragmentation as a function of resolution and radiation intensity.

The goal of this study is to identify fragmentation sites in collapsing metal-free atomic cooling haloes which are irradiated by a uniform LW background. As noted above these pristine haloes are expected to be ideal cradles for SMS formation if the $\mathrm{H}_{2}$ abundances can be suppressed. If fragmentation levels are reduced in the face of impacting LW radiation, as expected, this would be favourable for monolithic collapse of a single massive object. We probe this scenario in this work.

The paper is laid out as follows: in $\S 2$ we describe the model setup and the numerical approach used as well as introducing our star particle formulation; in $\S 3$ we describe the results of our numerical simulations; in $\S 4$ we discuss the importance of the results and present our conclusions.

Throughout this paper we assume a standard $\Lambda$ CDM cosmology with the following parameters (Planck Collaboration et al. 2014, based on the latest Planck data), $\Omega_{\Lambda, 0}=0.6817, \Omega_{\mathrm{m}, 0}=0.3183$, $\Omega_{\mathrm{b}, 0}=0.0463, \sigma_{8}=0.8347$ and $h=0.6704$. We further assume a spectral index for the primordial density fluctuations of $n=0.9616$.

\section{NUMERICAL FRAMEWORK}

In this study we have used the publicly available adaptive mesh refinement code Enzo ${ }^{1}$ to study the fragmentation properties of gas within haloes irradiated by a background LW field. Into Enzo we have added a new star particle type which we have dubbed Smartstar. We now describe both components.

\subsection{Enzo}

Enzo ${ }^{2}$ (Bryan et al. 2014) is an adaptive mesh refinement code ideally suited for simulations of the high redshift universe. Gravity in Enzo is solved using a fast Fourier technique (Hockney \& Eastwood 1988) which solves the Poisson equation on the root grid at each timestep. On subgrids, the boundary conditions are interpolated to the subgrids and the Poisson equation is then solved at each timestep. Dark matter is represented using particles, each particle is stored on the highest refinement grid available to it and thus the particle has the same timestep as the gas on that grid. The particle densities are interpolated onto the grid and solved at the same time as the gas potential. Enzo contains several hydrodynamics schemes to solve the Euler equation. We use the piecewise parabolic method which was originally developed by Berger \& Oliger (1984) and adapted to cosmological flows by Bryan et al. (1995). The PPM solver is an explicit, higher order accurate version of Godunov's method for ideal gas dynamics with a spatially third accurate piecewise parabolic monotonic interpolation scheme employed. A nonlinear Riemann solver is used for shock capturing. The method is formally second order accurate in space and time and explicitly conserves mass, linear

\footnotetext{
1 http://enzo-project.org/

2 Changeset:fedb30ff370b
} 

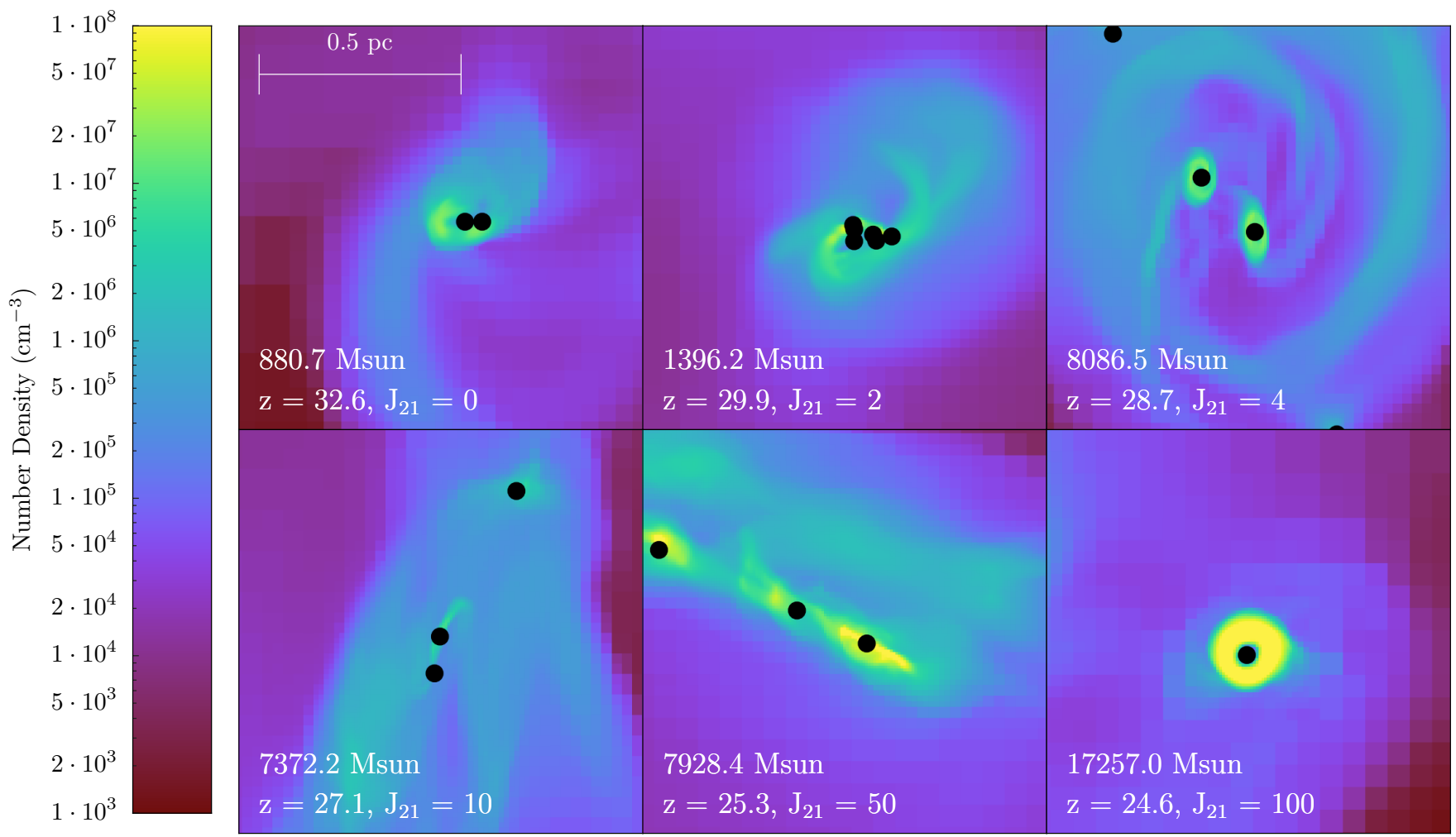

Figure 1. Projection of the number density through a 1 parsec volume surrounding the largest Smart Star particle in each case. The Lyman-Werner background radiation produced is varied from panel to panel from $0 \mathrm{~J}_{21}$ to $100 \mathrm{~J}_{21}$. Each realisation is therefore identical except for a different LW background field. As the radiation intensity is increased the collapse is delayed to later times as $\mathrm{H}_{2}$ is suppressed (note the redshift decreases with increasing LW intensity). This raises the Jeans mass of the halo and leads to larger Smart Star masses. The projections are created approximately 650 kyrs after the formation of the first Smart Star in each case.

momentum and energy making the scheme extremely useful for following the collapse of dense structures.

Chemistry is an important component is following the collapse of (ideal) gas. We use the Grackle ${ }^{3,4}$ (Smith et al. 2017) library to follow the evolution of ten individual species: $\mathrm{H}, \mathrm{H}^{+}, \mathrm{He}, \mathrm{He}^{+}, \mathrm{He}^{++}, \mathrm{e}^{-}, \mathrm{H}_{2}, \mathrm{H}_{2}^{+} \mathrm{H}^{-}$and $\mathrm{HeH}^{+}$. We adopt here the 26 reaction network determined by Glover (2015a) as the most appropriate network for solving the chemical equations required by gas of primordial composition with no metal pollution and exposed to an external radiation source. The network includes the most up-to-date rates as described in Glover \& Jappsen (2007); Glover \& Abel (2008); Glover \& Savin (2009); Coppola et al. (2011, 2012); Glover (2015a,b); Latif et al. (2015). The cooling mechanisms included in the model are collisional excitation cooling, collisional ionisation cooling, recombination cooling, bremsstrahlung and Compton cooling off the CMB.

\subsection{Simulation Setup}

All simulations are run within a box of $2 h^{-1}$ Mpc (comoving), the root grid size is $256^{3}$ and we employ three levels of nested grids. The grid nesting and initial conditions

\footnotetext{
3 https://grackle.readthedocs.org/

4 Changeset:482876c71f73
}

were created using MUSIC (Hahn \& Abel 2011). Within the most refined region (i.e. level 3) the dark matter particle mass is $\sim 103$ $\mathrm{M}_{\odot}$. In order to increase further the dark matter resolution of our simulations we split the dark matter particles according to the prescription of Kitsionas \& Whitworth (2002) and as described in Regan et al. (2015). We split particles centered on the position of the final collapse as found from lower resolution simulations within a region with a comoving side length of $43.75 \mathrm{~h}^{-1} \mathrm{kpc}$. Each particle is split into 13 daughter particles resulting in a final high resolution region with a dark matter particle mass of $\sim 8 \mathrm{M}_{\odot}$. The particle splitting is done at a redshift of 40 well before the collapse of the target halo. Convergence testing to study the impact of lower dark matter particle masses was discussed in Regan et al. (2015).

The baryon resolution is set by the size of the grid cells, in the highest resolution region this corresponds to approximately $0.48 h^{-1} \mathrm{kpc}$ comoving (before adaptive refinement). We vary the maximum refinement level (see Table 1) to explore the impact of resolution on our results. Refinement is triggered in Enzo when the refinement criteria are exceeded. The refinement criteria used in this work were based on three physical measurements: (1) The dark matter particle over-density, (2) The baryon over-density and (3) the Jeans length. The first two criteria introduce additional meshes when the over-density $\left(\frac{\Delta \rho}{\rho_{\text {mean }}}\right)$ of a grid cell with respect to the mean density exceeds 8.0 for baryons and/or DM. Furthermore, we set the MinimumMassForRefinementExponent parameter to -0.1 making the simulation super-Lagrangian and therefore reducing 


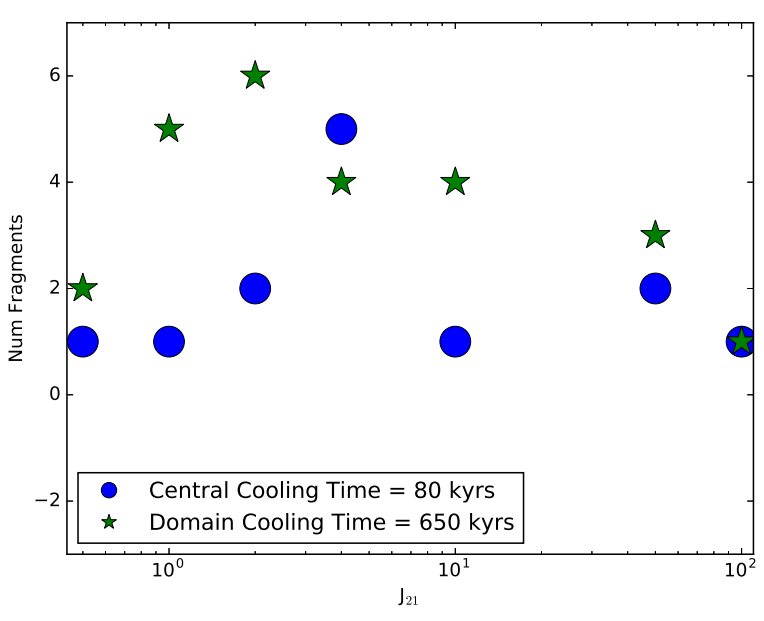

Figure 2. The number of fragments which form as the intensity of LymanWerner radiation is varied. Blue circles represent the number of fragments after a central cooling time of $80 \mathrm{kyrs}$ while green stars represent the number of fragments after a domain cooling time of 650 kyrs. The resolution in each case is set at $\Delta x \sim 4 \times 10^{-3} \mathrm{pc}$ (physical).

the threshold for refinement as higher densities are reached. For the final criteria we set the number of cells per Jeans length to be 32 in these runs.

We use 16 levels of refinement as our fiducial refinement level. This corresponds to a minimum cell size of $\Delta x \sim 0.01$ pc/h comoving $\left(\sim 6 \times 10^{-3} \mathrm{pc}\right.$ at $\left.\mathrm{z} \sim 30\right)$. We set the effective temperature of the background radiation field to $\mathrm{T}_{\text {eff }}=30000$ $\mathrm{K}$. This background temperature suitably models the spectrum of a population of young stars (Wolcott-Green \& Haiman 2012; Sugimura et al. 2014; Latif et al. 2015). The effective temperature of the background is important as the radiation temperature determines the dominant photo-dissociation reaction set in the irradiated halo. This in turn leads to a value of $\mathrm{J}_{\text {crit }}$ - the flux above which complete isothermal collapse of the irradiated halo is observed due to the complete suppression of $\mathrm{H}_{2}$. The actual value of $\mathbf{J}_{\text {crit }}$ depends on the nature of the source spectrum (Shang et al. 2010; Sugimura et al. 2014; Agarwal \& Khochfar 2015).

Agarwal et al. (2016) proposed that the $\mathbf{J}_{\text {crit }}$ needed from a given stellar population modelled using realistic stellar spectra can vary widely over 2-3 orders of magnitude. They argue that in an external pristine atomic cooling halo, $\mathrm{DCBH}$ formation is better parameterised by using a critical curve in the $\mathrm{H}_{2}$ and $\mathrm{H}^{-}$photo-destruction rate parameter space (further confirmed by (Wolcott-Green et al. 2017)). While our choice of $\mathrm{T}_{\text {eff }}=30000 \mathrm{~K}$ falls well within the range advocated by these studies, including a realistic source spectrum derived from population synthesis models is beyond the scope of the current work and remains to be explored in a future study. It should also be noted that Sugimura et al. (2014) found that $\mathbf{J}_{\text {crit }}$ is only very weakly dependent on the nature of the source spectrum in tension with the results of both Agarwal et al. (2016) and (Wolcott-Green et al. 2017), however they explored a somewhat smaller parameter space.

\subsection{Smart Stars}

As the gas density increases in high density regions hydro codes, including En zo, require a method to convert the high density gas into stars in many cases. This is done to allow gas which has reached the maximum allowed refinement level of the simulation and for which further collapse is being artificially suppressed through artificial pressure support to be dealt with. In this case it can often be prudent to introduce particles into the calculation to mimic the act of real star formation. It should however be noted that in many cases there is only a loose correspondence between the numerical particle and resolving actual star formation. Depending on the level of resolution, primarily, the numerical particle may represent a single star or an entire cluster of stars. In our study here the numerical particle introduced will be a proxy for star formation (fragmentation) sites and represent only regions in which we expect star formation to be likely to occur. Star (sometimes also known as sink) particles were initially introduced into grid codes by Krumholz et al. (2004). The Smartstar implementation used here also follows this model with some modifications. We employ the same model for gas accretion as Krumholz et al. (2004) for our star particles using a fixed accretion radius of four cells and accreting gas based on the Bondi-Hoyle prescription. However, while Krumholz et al. (2004) use only two criteria to evaluate the formation of sink particles; that the gas is at the highest refinement level and that the cell density exceeds the Jeans density (see equation 6 in Krumholz et al. (2004)) we also employ additional sink particle formation criteria. Similar to the prescriptions described by Federrath et al. (2010) we only form sink particles when the following criteria are met:

(i) The cell is at the highest refinement level

(ii) The cell exceeds the Jeans Density

(iii) The flow around the cell is converging along each axis

(iv) The cooling time of the cell is less than the freefall time

(v) The cell is at the minimum of the gravitational potential

We calculate the gravitational potential in a region of twice the Jeans length around the cell. We experimented with also including the additional conditions relating to the gas boundedness and the Jeans instability test (see Federrath et al. (2010) for more details). However, we found that these additional tests were sub-dominant compared to the criteria noted above and so in the interest of optimisation we did not include them.

Once a Smartstar is formed it can accrete gas within its accretion radius (4 cells) and it can merge with other Smartstar particles. In this study we are focused only on ascertaining the degree of fragmentation experienced in haloes as a function of Lyman-Werner radiation. The SmartStar particle type contains algorithms for different accretion modes and feedback modes as well as the ability to differentiate between normal PopIII star formation and supermassive star formation based on accretion rates. However, we do not employ these more sophisticated accretion or feedback algorithms here and instead leave those for an upcoming study. Here as noted previously we focus only on identifying fragmentation sites in collapsing atomic cooling haloes. In Table 1 we list the simulation parameters and some of the fundamental Smartstar parameters and results.

\section{RESULTS}

\subsection{The onset of fragmentation as a function of LW intensity}

In Figure 1 we show the central 1 pc of the simulations with LW intensities of $0 \mathrm{~J}_{21}{ }^{5}, 2 \mathrm{~J}_{21}, 4 \mathrm{~J}_{21}, 10 \mathrm{~J}_{21}, 50 \mathrm{~J}_{21}, 100 \mathrm{~J}_{21}$. Each

\footnotetext{
${ }^{5} \mathrm{~J}_{21}$ is defined as $10^{-21} \mathrm{erg} \mathrm{cm}^{-2} \mathrm{~s}^{-1} \mathrm{~Hz}^{-1} \mathrm{sr}^{-1}$
} 


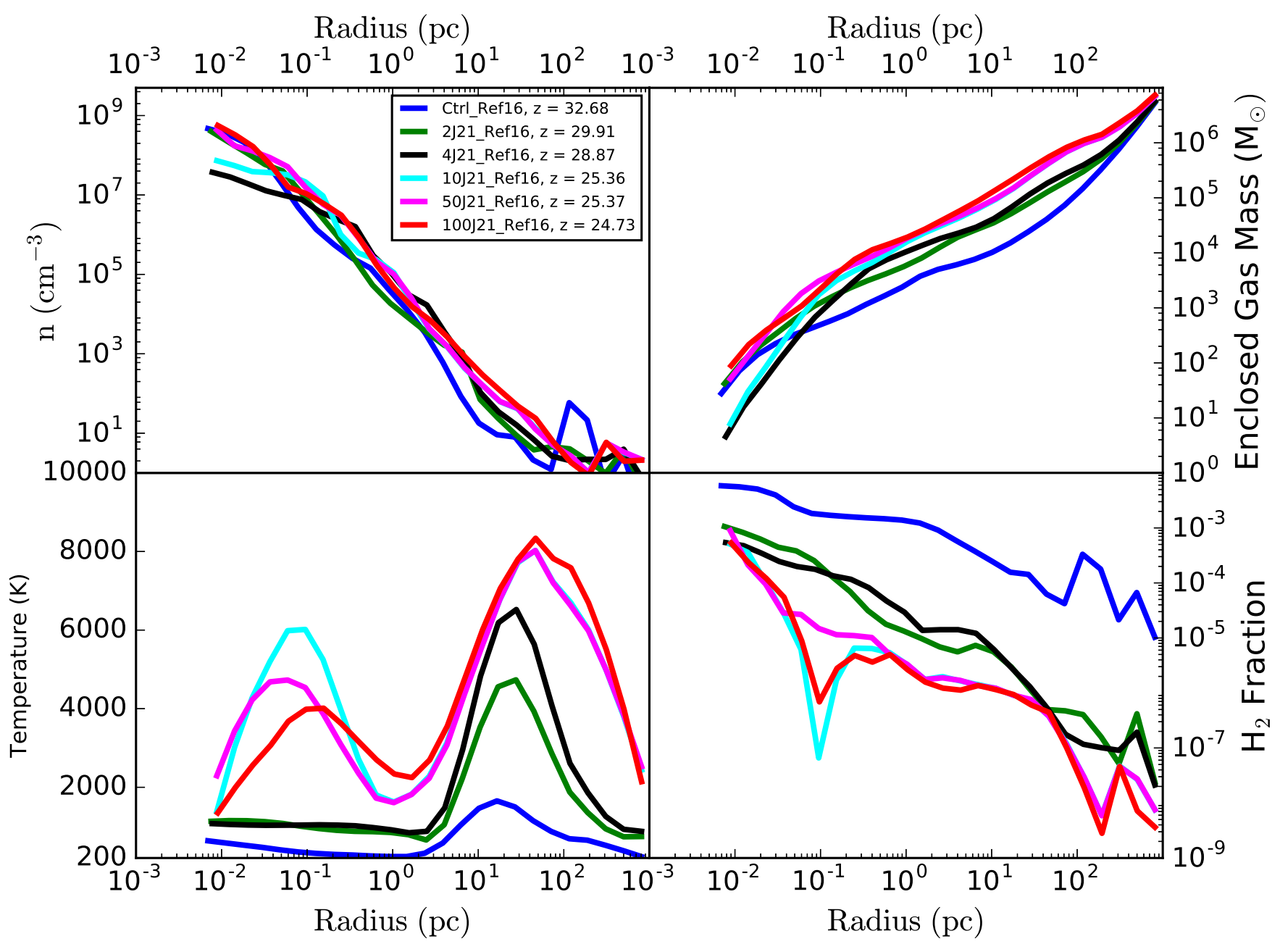

Figure 3. Radial profiles of the gas just prior to the formation of the first SmartStar particle. The profiles are for realisations with the same maximum resolution (refinement level of 16 , see Table 1) but for varying $\mathrm{LW}$ intensities. For $\mathrm{LW}$ intensities of $\mathrm{J} \gtrsim 10 \mathrm{~J}_{21}$ there is a noticeable switch in the temperature of the gas (see bottom left panel). For these larger intensities the gas is switching to the atomic cooling regime and as we will see fragmentation becomes suppressed.

panel has an identical maximum refinement level of 16 levels and hence an equivalent minimum cell size in comoving units. The projection is made 650 kyrs after the first Smart Star forms. Each realisation shows the formation of multiple fragments within the collapsing gas with the exception of the halo exposed to a radiation level of $100 \mathrm{~J}_{21}$. In the final halo of Figure 1 (i.e. bottom right panel) only a single fragment forms at the very centre of the halo and is surrounded by a dense accretion disk (though mild fragmentation is also observed in this realisation at higher resolution as we will see). The mass of the fragments show a general trend of increasing mass with increasing radiation level as expected since the Jeans mass increases in each case due to the suppression of $\mathrm{H}_{2}$.

In the bottom left of each panel in Figure 1 we give the mass of the most massive Smart Star at this time. We do not directly associate this mass with the final mass of the star that forms since further fragmentation may well, and does (see Figure 5), occur below this maximum resolution scale. That is not the goal in this study. Instead we are focused on identifying regions within the collapsing gas cloud which are likely to experience fragmentation and likely to foster star formation. Interestingly what we see is that, initially, as the LW intensity increases, there is a noticeable increase in the number of fragmentation sites.

In Figure 2 we have plotted the number of fragments as a function of the background LW intensity for our fiducial refinement level of 16. We choose two different times at which to examine the number of fragments. We calculate the cooling time in a given region, $t_{c o o l}$, as the average cooling time in a region. The cooling time is the time after which the first Smartstar forms that we expect an actual star to form within the fragmentation site. The coarser the resolution the longer this time will be. At the other extreme with infinite resolution the fragmentation site would be the actual star and $t_{\text {cool }}$ would be zero. In order to account for our limited resolution we use the cooling time as a estimate of the collapse of the gas within the fragmentation site into stars a process which occurs well below the grid scale.

We initially select each cell and calculate its Jeans length. We then calculate the average cooling time in a sphere surrounding that cell with radius its Jeans length. We then loop over all cells in this manner calculating the cooling over multiple regions to find the cooling time over a given domain. The equation for the domain 


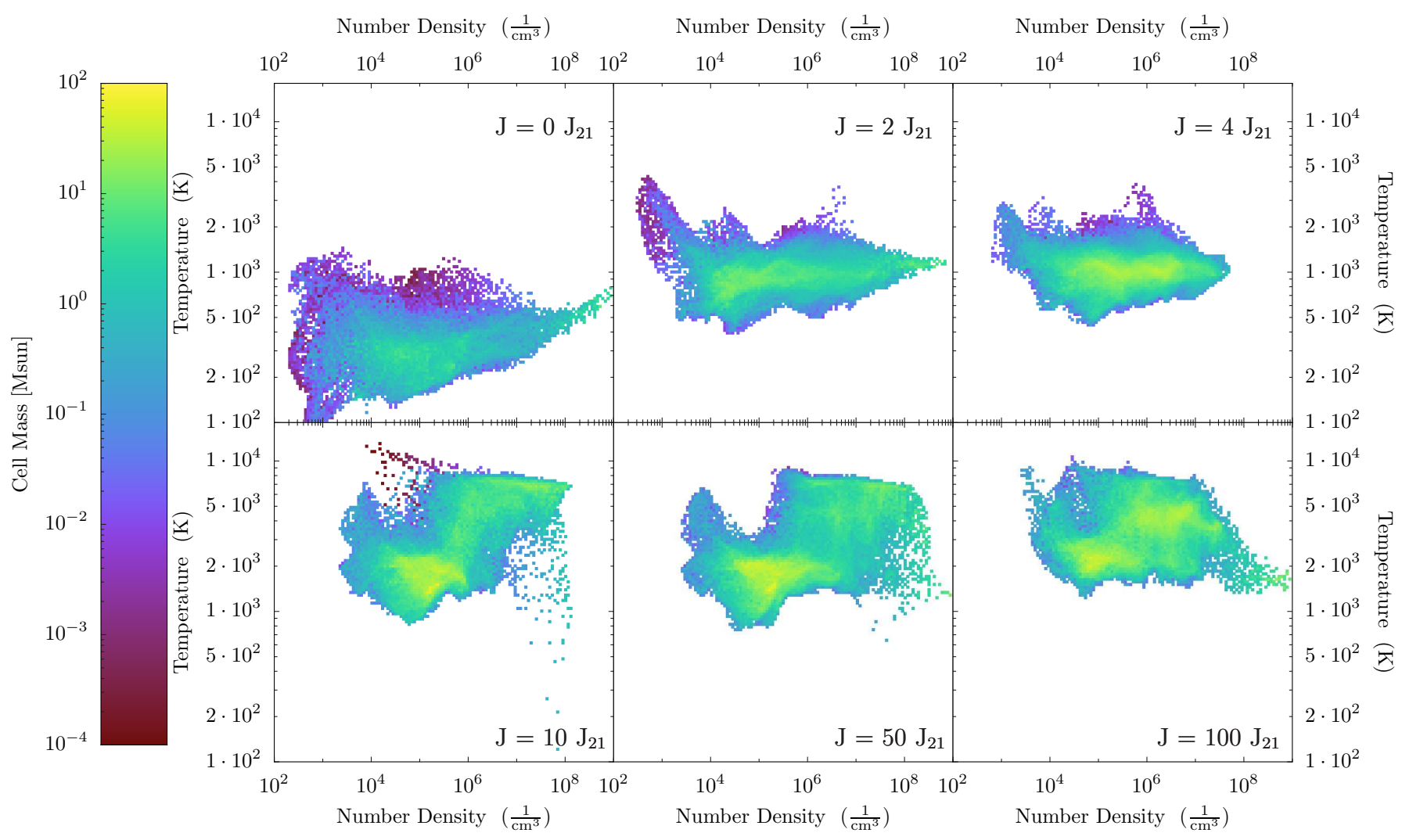

Figure 4. The thermodynamic state of the gas as a function of LW intensity at fixed resolution (maximum refinement 16 ). As the LW intensity increases the $\mathrm{H}_{2}$ fraction is suppressed altering the cooling function of the gas. Initially $\left(\mathrm{J}=0 \mathrm{~J}_{21}\right)$ the gas remains cool as the collapse takes hold, however, as the intensity increases the gas moves onto the atomic cooling track with cooling regulated by atomic cooling at closer to $8000 \mathrm{~K}$.

cooling time, $\tau_{\text {cool }}$, is then given by:

$$
\begin{aligned}
t_{\text {cool }} & =<\sum_{i=0}^{N_{\text {cell }}} t_{\text {cool }, i}^{J_{v}}> \\
\tau_{\text {cool }} & =\frac{\sum_{i=0}^{N_{\text {cell }}} t_{\text {cool }} \rho_{i}}{\sum_{i=0}^{N_{\text {cell }} \rho_{i}}}
\end{aligned}
$$

where $N_{\text {cell }}$ is the number of cells in the domain and $J_{V}$ is the Jeans volume (i.e. the sphere surrounding the cell $\mathrm{i}$ of radius the jeans length of that cell) and $t_{\mathrm{cool}, i}^{J_{V}}$ is the average cooling time of the gas within that cooling sphere. We then sum over all cooling spheres (i.e. all cells) and taking the average cooling time within each sphere as the cooling time for that sphere. We further weight the contribution of each cell in the calculation by it's density with the highest density cells (those with the shortest cooling times) having a larger contribution. Low density cells then do not have an appreciable contribution to the calculation.

We choose two different domains. In the first instance we choose the Jeans length of the maximum density cell and examine all cells within a sphere of radius the Jeans length surrounding that cell $\left(J_{L} \sim 0.03 \mathrm{pc}\right)$. That leads to an average cooling time of approximately 80 kyrs for the fiducial runs (i.e. those with a maximum refinement level of 16). The second region we choose is the entire computational domain. We found through experimentation that the cooling time of the domain saturates after the region exceeds approximately $10 \mathrm{kpc}$ as outside of this region the densities become too low and so those cells do not contribute significantly to the computation of the average cooling time. We find the aver- age cooling time in the total computational domain corresponds to approximately 650 kyrs. The cooling time will be somewhat dependent on resolution as well. As higher densities are resolved we are able to better resolve the actual star formation timescale and hence our proxy for the star formation timescale (here estimated using the cooling time) drops. We fix the computation to only calculate the cooling time for the fiducial refinement level as a benchmark.

The first time ( 80 kyrs) corresponds to roughly when we expect the first actual star to form after the Smartstar formation has been triggered. This will also correspond to the time at which we expect radiation feedback effects to begin to impact subsequent fragmentation. The second time is the other extreme and is the maximum time it could take the surrounding gas to cool and initiate widespread fragmentation. These two timescales should then bracket the actual fragmentation timescale - which is unknown and will depend on specific environmental conditions which are either not resolved by our simulation or not included. In determining the fragmentation scale the Jeans length of the gas must be adequately resolved. The Jeans length (and cooling time/length) depend sensitively on the temperature of the gas. In the $0 \mathrm{~J}_{21}$ case the Jeans length is of order $J_{L} \sim 0.007 \mathrm{pc}$ at the centre of the collapse while it increases to approximately $J_{L} \sim 0.04$ pc for the cases with radiation. For our fiducial runs, $\Delta x \sim 0.004 \mathrm{pc}$, and so we are not well resolving fragmentation sites for the $0 \mathrm{~J}_{21}$ case. The number of fragmentation sites for the $0 \mathrm{~J}_{21}$ case can only be therefore taken as lower limits. For the cases with radiation, which is the goal of this study, we are resolving the Jeans length reasonably well. Nonetheless we include the $0 \mathrm{~J}_{21}$ case for the case of completeness. 


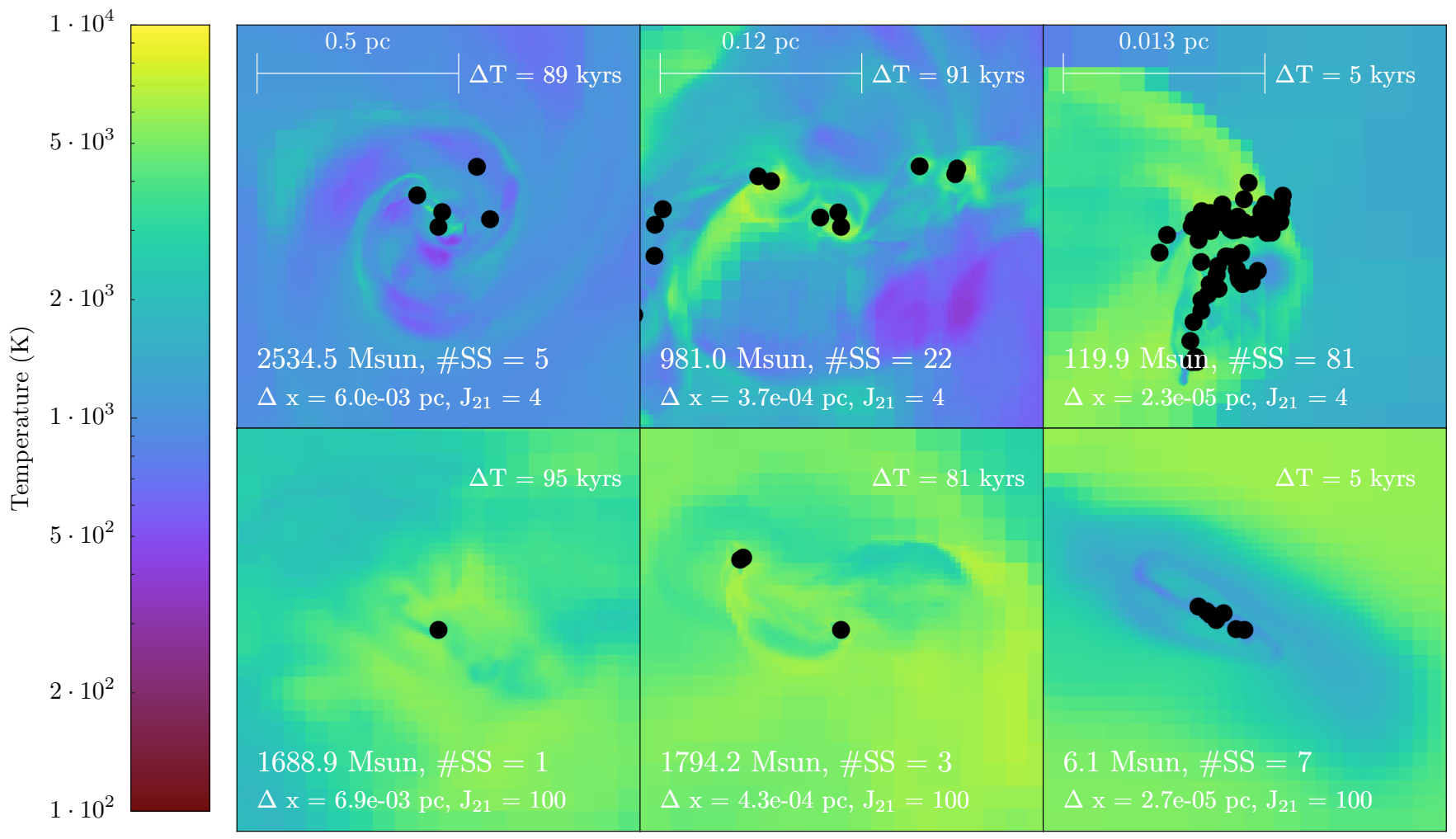

Figure 5. The fragmentation as the resolution is changed for haloes exposed to LW intensities of $4 \mathrm{~J}_{21}$ and $100 \mathrm{~J}_{21}$ respectively. The time of the snapshot, $\Delta T$, is given in the top right corner of each panel and represents the age of the most massive Smartstar particle. Also given is the mass of the most massive particle, the number of fragments recorded, the minimum spatial scale resolved and the LW intensity. The width of the panel decrease from left to right as indicated by the scale line, the scale is decreases so that the individual fragments are visible as the resolution is increased.

For the case of $\mathrm{J}=4 \mathrm{~J}_{21}$ the number of fragmentation sites is 5 after 80 kyrs. It is only when the background is sufficiently strong that it can effect the detailed chemistry of the gas - we then see a reduction in the number of fragmentation sites. For the highest intensity case probed here $\left(\mathrm{J}=100 \mathrm{~J}_{21}\right)$ the number of fragmentation sites is one both after $80 \mathrm{kyrs}$ and after $650 \mathrm{kyrs}$. If we focus on the trend from $2 \mathrm{~J}_{21}$ to $100 \mathrm{~J}_{21}$ we see the number of fragmentation sites decrease as a function of increasing radiation. While there is also a drop from $1 \mathrm{~J}_{21}$ to $0 \mathrm{~J}_{21}$ this is likely due to a lack of resolution in the fiducial case and the fragmentation levels for the $0 \mathrm{~J}_{21}$ are best treated as a lower limit.

In Figure 3 we plot the radial profiles of the gas as a function of radius for the realisation at the same refinement level (Ref16) and varying background intensities. The plots are made just before the first Smart Star forms in each realisation. The densities reached are approximately the same in each case since the maximum refinement level is identical and the densities are close to those at which Smartstar formation is triggered. Note that the times (redshifts) are different in each case as the LW background delays the onset of cooling within the halo. Note also how the enclosed mass, $\mathrm{H}_{2}$ fraction and temperature vary according to the LW intensity. For the highest LW backgrounds the temperature is clearly higher in the central parts of the halo. These thermodynamic differences effect the fragmentation rates as we saw in Figure 2. To further emphasise the temperature and associated thermodynamic differences we show the phase profiles for each realisation in Figure 4. As the LW intensity increases we clearly see the state of the gas change reflecting the chemical changes. As the intensity increases up to and above $10 J_{21}$ we see the gas transition from the molecular to the atomic cooling regime with a significant mass fraction of the gas above approximately $2000 \mathrm{~K}$. This transition and the thermodynamic consequences of it are reflected in the decrease in the level of fragmentation we observe. The increased temperature of a significant fraction of the gas results in a larger Jeans mass and less fragmentation for the most highly irradiated haloes.

\subsection{The dependence of fragmentation on resolution}

In Figure 5 we plot the fragmentation as a function of resolution for two different values of $\mathrm{LW}$ intensity $-4 \mathrm{~J}_{21}$ and $100 \mathrm{~J}_{21}$. In the left hand column the maximum refinement level is set to 16 ( $\Delta x \sim 6 \times 10^{-3} \mathrm{pc}$ ), in the middle column the maximum refinement level is increased to $20\left(\Delta x \sim 4 \times 10^{-4} \mathrm{pc}\right)$ and in the right hand column the maximum refinement level is increased to 24 ( $\Delta x \sim 3 \times 10^{-5} \mathrm{pc}$ ). For the left and middle column the snapshots are plotted at approximately 80 kyrs (i.e. the cooling time with a Jeans length of the gas for the Ref16 runs) while for the right hand column the short dynamical times make extending the runs to those times prohibitive and instead we plot them at 5 kyrs instead (cf. Stacy et al. (2016)).

The projection is made using the temperature field to illustrate the changing chemical conditions as a function of LW intensity. Note that the spatial scale varies between columns from left to right. We centre on the most massive Smartstar in each case and as the resolution increases we are probing fragmentation deeper and 


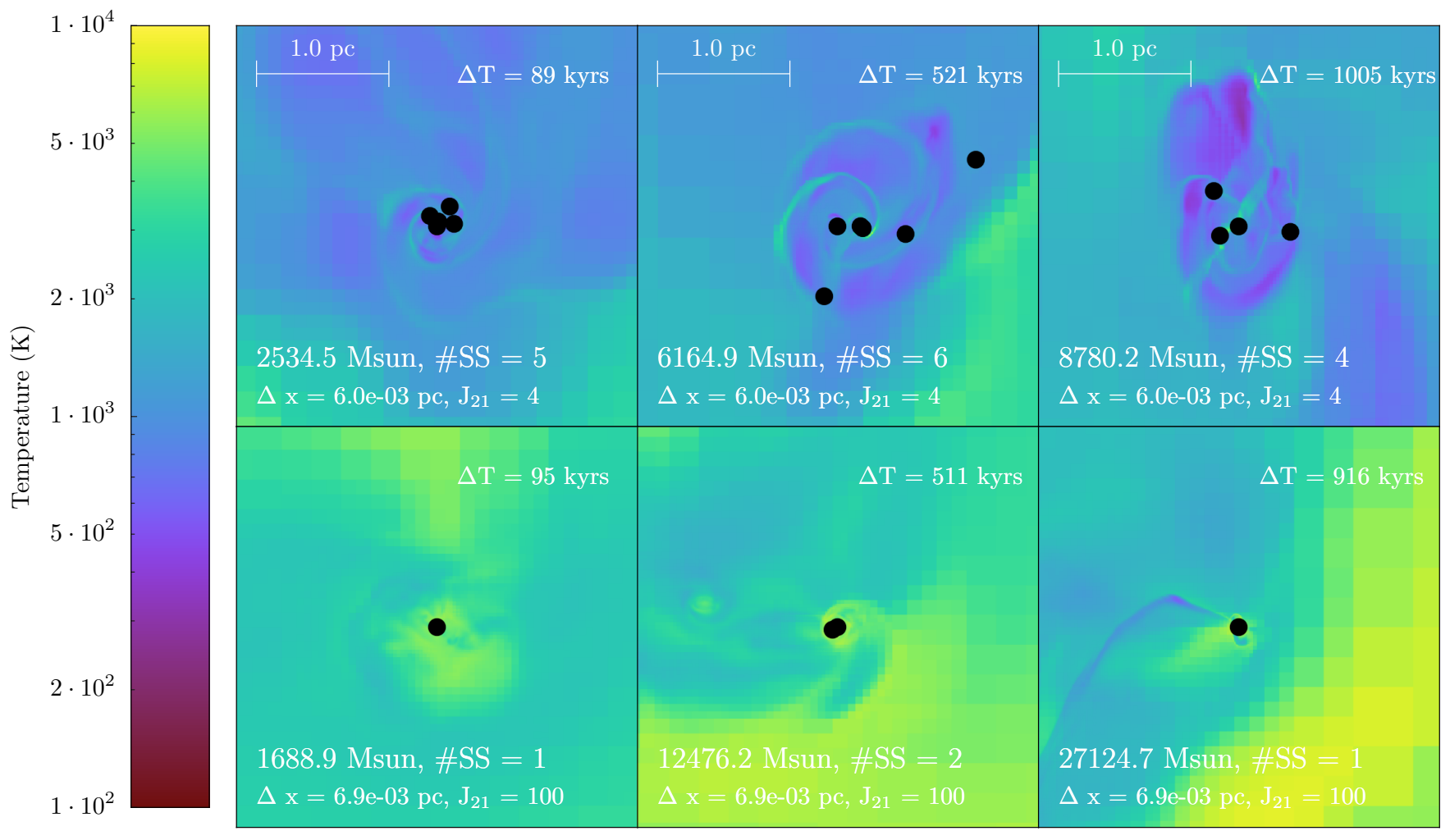

Figure 6. The fragmentation for different snapshots for haloes exposed to LW intensity of $4 \mathrm{~J}_{21}$ and $100 \mathrm{~J}_{21}$ respectively. In the left hand column the output is approximately $90 \mathrm{kyrs}$ after the formation of the first Smart Star, in the middle column approximately 500 kyrs and the right hand column approximately 1 Myr. While some mild fragmentation is observed in the $4 \mathrm{~J}_{21}$ case it is essentially absent in the $100 \mathrm{~J}_{21}$ case. The time of the snapshot, $\Delta T$, is given in the top right corner of each panel and represents the age of the largest Smart Star particle. Also given is the mass of the most massive particle, the number of fragments recorded, the minimum spatial scale resolved and the LW intensity.

deeper inside individual fragmentation sites. As the resolution is increased we see that the $4 \mathrm{~J}_{21}$ realisation shows increased fragmentation, with the colder gas and subsequently smaller Jeans masses likely facilitating the fragmentation. This is in contrast with the bottom row where the $100 \mathrm{~J}_{21}$ intensities show comparatively little fragmentation for similar scales. The right hand column shows the most significant fragmentation demonstrating the most complex structures exist at the smallest scales. Again, however, the 100 $\mathbf{J}_{21}$ intensities show over an order of magnitude less fragmentation compared to the $4 \mathrm{~J}_{21}$ intensities at comparable times. The prohibitively short Courant times associated with the highest resolution runs (even allowing for the fact that a portion of the high density gas is tied up in particles) prevents us from evolving the highest resolution simulations for a large number of dynamical times. However, what is clear is that the level of fragmentation is greatly reduced at the smallest scales as the LW intensity is increased.

\subsection{Further evolution of fragmentation}

Finally in Figure 6 we explore the time evolution of the realisations with LW intensity of $4 J_{21}$ and $100 J_{21}$ respectively. It should be noted here again that we do not include the effects of radiation or accretion feedback in these simulations and therefore we are neglecting some important physical processes which will effect the levels of fragmentation as star formation proceeds. Additionally, the formation of one Smart Star, may impact physically, on the formation of a subsequent Smart Star. This would be particularly important if the SmartStar releases ionising radiation. For the case of SMS formation with accretion rates around $0.1 \mathrm{M}_{\odot} \mathrm{yr}^{-1}$ the bloated stellar radii of SMSs lead to effective surface temperatures of approximately $6000 \mathrm{~K}$. With these, relatively low, effective temperatures the radiation is emitted predominantly in the infrared meaning that it's effect may be quite mild and not impact surrounding fragmentation sites. Therefore, for the high external flux cases where SMS formation is expected, the formation of one SmartStar is not expected to negatively (or positively) impact subsequent SMS formation. If, however, smaller POPIII stars form instead then the ionisation front may become important. However, a recent study by Chon et al. (2017) shows that the ionisation from around POPIII stars which form in close proximity to SMSs have small HII regions which are closely bound to the star and do not have significant negative impacts at least in the first 100,000 years. However, since we do not take these feedback effects into account here, we simply outline the evolution of fragmentation in the absence of these effects. In Figure 6 we see that initially (left hand column) the Smartstar particles are quite clustered (there is only particle for the $100 \mathrm{~J}_{21}$ case). As the simulation proceeds up to 1 Myr (after which the first SmartStar particle would be expected to go supernova) the SmartStar fragmentation sites in the $4 \mathbf{J}_{21}$ spread out and lie approximately $1 \mathrm{pc}$ apart. For the $100 \mathrm{~J}_{21}$ case the collapse remains completely monolithic. We can also see that for the $4 \mathrm{~J}_{21}$ case the surrounding gas remains comparatively cold compared to the $100 \mathrm{~J}_{21}$ case, the temperature of the gas in the 4 $\mathrm{J}_{21}$ case is likely to stimulate fragmentation and hence the increased 
fragmentation in the lower LW intensity case is not surprising. The main finding is that the transition to the atomic cooling regime in the $100 \mathrm{~J}_{21}$ case leads to and sustains complete monolithic collapse.

\subsection{Comparison to previous studies in the literature}

L14b and Latif \& Volonteri (2015) (L15b) performed similar studies to that performed here. In L14b their goal was to identify the mass scale of stars formed when exposed to a moderate LW background and in L15b they investigated the accretion rates on sink particles in the case of non-isothermal collapse. We will focus our comparison with the first work - L14b - as it most closely corresponds to our study. In L14b they investigated the flux impinging on two separate haloes with LW backgrounds of $10 \mathrm{~J}_{21}, 100 \mathrm{~J}_{21}$ and $500 \mathrm{~J}_{21}$. Unlike the study performed here they did not systematically vary their resolution and did not explore lower LW background cases (i.e. below $10 \mathrm{~J}_{21}$ ). Their sink particle prescription was based on that of Wang et al. (2010) which is broadly similar to the prescription we employed although their accretion radius was significantly larger than ours $(\sim 32$ cells compared to our 4 cells). Nonetheless our results are broadly consistent for the relatively high flux cases of $\mathbf{J}=10 \mathrm{~J}_{21}$ and 100 $\mathrm{J}_{21}$ where our two studies overlap. The main differences being that we form more sinks for the very high resolution tests $\left(\delta_{x} \sim\right.$ $1 \mathrm{AU}$ ) but with smaller masses. L14b find that one to two sinks form in their very high resolution cases. We find, at comparable resolution to their study, between 3 and 7 sinks have formed. Our higher values are due to the fact that we have a much smaller accretion radius and hence less merging (since the merging radius is identical to the accretion radius in both implementations). Our mass scales therefore also differ somewhat, L14b have masses of $7337 \mathrm{M}_{\odot}$ and $2592 \mathrm{M}_{\odot}$ for their haloes, for the $\mathrm{J}=100 \mathrm{~J}_{21}$ after 10 kyr. In our work we see sink particles with maximum masses of $1794 \mathrm{M}_{\odot}$ (after $80 \mathrm{kyr}$ ) and $6 \mathrm{M}_{\odot}$ (after $5 \mathrm{kyr}$ ). Our decreased masses can be attributed to our significantly smaller accretion radius. Choosing the correct accretion radius is non-trivial and different prescriptions for the accretion/merging radius will lead to different results as noted recently by Becerra et al. (2017) in a similar context. Nonetheless, the studies give broadly the same result with differences attributable to slightly different subgrid implementations. Since their study did not investigate the lower flux cases the degree of fragmentation suppression is not available from their study.

Comparing to the results of Stacy et al. (2016), which is for the zero flux case, we achieve similar resolution and similar results to their very high resolution runs. While they investigate the build up of a PopIII IMF we focus on simply identifying fragmentation sites rather than individual star formation sites. Encouragingly their fragmentation levels are similar to ours (comparing our $4 \mathbf{J}_{21}$ case to their fiducial $\left(0 \mathrm{~J}_{21}\right)$ case $)$. After approximately $5 \mathrm{kyrs}$ the ionisation front from the first star to form has slowed the accretion rates and subsequent star formation in their simulations. They find that of the order of 100 sink particles have formed after $5 \mathrm{kyr}$ compared to our 81 sink particles. It is encouraging to see that given our resolution levels are comparable that we achieve similar fragmentation levels for the low flux cases when the dominant chemistry is still due to $\mathrm{H}_{2}$.

\section{SUMMARY \& DISCUSSION}

We investigated here the degree of fragmentation of atomic cooling haloes as a function of both the strength of the LW background and as a function of resolution. The degree of fragmentation within irradiated atomic cooling haloes is an important consideration in understanding the zero age main sequence masses of supermassive stars (e.g. Hosokawa et al. 2013b; Woods et al. 2017; Haemmerlé et al. 2017) that are candidates for the supermassive black holes that exist as high redshift quasars (Haiman 2006; Regan \& Haehnelt 2009a; Volonteri 2010).

Haloes that are exposed to high Lyman Werner backgrounds have delayed collapse times and larger Jeans masses enabling them to form larger objects. However, if fragmentation of the gas is prevalent the final masses of the (super)massive stars is likely to be greatly reduced. The thermodynamic conditions of the gas is therefore critical in estimating the initial and (by accounting for mass loss) the final masses of these objects.

We find that haloes exposed to a $\mathrm{LW}$ background of $\mathbf{J} \gtrsim 10 \mathrm{~J}_{21}$ experience progressively less fragmentation than haloes exposed to lower LW backgrounds. Haloes exposed to a LW background of $\mathbf{J} \gtrsim 1_{21}$ have a significant fraction of their gas pushed onto the atomic cooling track (see Figure 4) and are much less prone to fragmentation. The transition to the atomic cooling track is likely then to be the most stringent indicator of fragmentation levels. The increased temperature of the gas in the centre limits the degree of fragmentation significantly and for the highest irradiation levels examined here fragmentation was eradicated almost entirely.

Comparing to the results of Stacy et al. (2016) we achieve similar resolution and similar results to their very high resolution runs. While they investigate the build up of a PopIII IMF we focus on simply identifying fragmentation sites rather than individual star formation sites. Encouragingly their fragmentation levels are similar to ours (comparing our $4 \mathbf{J}_{21}$ case to their fiducial $\left(0 \mathbf{J}_{21}\right)$ case). After approximately 5 kyrs the ionisation front from the first star to form has slowed the accretion rates and subsequent star formation in their simulations. Given our resolution levels are comparable it suggests that our assertion that once the gas chemistry switches to the atomic cooling regime fragmentation is likely to be strongly suppressed which is something they did not investigate.

We note also that any mechanism which suppresses $\mathrm{H}_{2}$ formation and forces a transition to the atomic cooling track is likely to achieve a similar outcome. For example in the simulations conducted by Schauer et al. (2017), collapsing haloes are exposed to streaming velocities of up to $18 \mathrm{~km} \mathrm{~s}^{-1}$ at $\mathrm{z}=200$. The streaming velocities act in a similar way to a $\mathrm{LW}$ background by suppressing $\mathrm{H}_{2}$ production and hence impacting the gas cooling. The thermodynamic state of the gas is therefore effected in a similar way to a LW background and hence for haloes exposed to large streaming velocities fragmentation is also likely to be suppressed. Similarly, dynamical heating effects (e.g. Yoshida et al. 2003) combined with mild LW radiation from nearby galaxies as recently identified by Wise et al. (2017)(in prep) would likely not be effected by vigorous fragmentation and would in fact be ideal candidates for a single monolithic collapse.

In summary we find that haloes exposed to LW radiation experience both delayed collapse and suffer from decreased fragmentation. Once the gas in the core exceeds approximately $2000 \mathrm{~K}$, and has transitioned chemically to predominantly atomic line cooling, then fragmentation is almost entirely suppressed and conditions becomes ideal for monolithic collapse. In future work we will explore 
the impact of feedback from the collapsing protostar and the subsequent transition to a (massive) black hole seed.

\section{ACKNOWLEDGEMENTS}

J.A.R and T.P.D thank David Hubber, Peter Johansson and Bhaskar Agarwal for useful discussion on both the sink particle implementation and direction of the study. J.A.R. acknowledges the support of the EU Commission through the Marie Skłodowska-Curie Grant - "SMARTSTARS" - grant number 699941. Computations described in this work were performed using the publicly-available Enzo code (http://enzo-project.org), which is the product of a collaborative effort of many independent scientists from numerous institutions around the world. Their commitment to open science has helped make this work possible. The freely available astrophysical analysis code YT (Turk et al. 2011) was used to construct numerous plots within this paper. The authors would like to extend their gratitude to Matt Turk et al. for an excellent software package. J.A.R. would like to thank Lydia Heck and all of the support staff involved with Durham's COSMA4 and DiRAC's COSMA5 systems for their technical support. This work was supported by the Science and Technology Facilities Council (grant numbers ST/L00075X/1 and RF040365). This work used the DiRAC Data Centric system at Durham University, operated by the Institute for Computational Cosmology on behalf of the STFC DiRAC HPC Facility (www.dirac.ac.uk). This equipment was funded by BIS National E-infrastructure capital grant ST/K00042X/1, STFC capital grant ST/H008519/1, and STFC DiRAC Operations grant ST/K003267/1 and Durham University. DiRAC is part of the National E-Infrastructure. The authors also wish to acknowledge the SFI/HEA Irish Centre for High-End Computing (ICHEC) for the provision of computational facilities and support. Finally, we thank the anonymous referee for comments which improved both the quality and clarity of the manuscript.

\section{REFERENCES}

Abel T., Bryan G. L., Norman M. L., 2000, ApJ, 540, 39

Agarwal B., Khochfar S., 2015, MNRAS, 446, 160

Agarwal B., Khochfar S., Johnson J. L., Neistein E., Dalla Vecchia C., Livio M., 2012, MNRAS, 425, 2854

Agarwal B., Davis A. J., Khochfar S., Natarajan P., Dunlop J. S., 2013, MNRAS, 432, 3438

Agarwal B., Dalla Vecchia C., Johnson J. L., Khochfar S., Paardekooper J. P., 2014, MNRAS, 443, 648

Agarwal B., Smith B., Glover S., Natarajan P., Khochfar S., 2016, MNRAS, 459, 4209

Alvarez M. A., Wise J. H., Abel T., 2009, ApJ, 701, L133

Bate M. R., Burkert A., 1997, MNRAS, 288, 1060

Becerra F., Marinacci F., Inayoshi K., Bromm V., Hernquist L. E., 2017, ArXiv e-prints:1702.03941

Begelman M. C., Volonteri M., Rees M. J., 2006, MNRAS, 370, 289

Berger M. J., Oliger J., 1984, Journal of Computational Physics, 53, 484

Boss A. P., Bodenheimer P., 1979, ApJ, 234, 289

Bromm V., Coppi P. S., Larson R. B., 1999, ApJ, 527, L5

Bryan G. L., Norman M. L., Stone J. M., Cen R., Ostriker J. P., 1995, Computer Physics Communications, 89, 149
Bryan G. L., Norman M. L., O'Shea B. W., Abel T., Wise J. H., Turk M. J., The Enzo Collaboration, 2014, ApJS, 211, 19

Burkert A., Bodenheimer P., 1993, MNRAS, 264, 798

Burkert A., Bate M. R., Bodenheimer P., 1997, MNRAS, 289, 497

Chon S., Hosokawa T., Yoshida N., 2017, ArXiv eprints: 1711.05262

Clark P. C., Glover S. C. O., Smith R. J., Greif T. H., Klessen R. S., Bromm V., 2011, Science, 331, 1040

Coppola C. M., Longo S., Capitelli M., Palla F., Galli D., 2011, ApJS, 193, 7

Coppola C. M., D'Introno R., Galli D., Tennyson J., Longo S., 2012, ApJS, 199, 16

Fan X., Carilli C. L., Keating B., 2006, ARA\&A, 44, 415

Federrath C., Banerjee R., Clark P. C., Klessen R. S., 2010, ApJ, 713, 269

Glover S. C. O., 2015a, MNRAS, 451, 2082

Glover S. C. O., 2015b, MNRAS, 453, 2901

Glover S. C. O., Abel T., 2008, MNRAS, 388, 1627

Glover S. C. O., Jappsen A. K., 2007, ApJ, 666, 1

Glover S. C. O., Savin D. W., 2009, MNRAS, 393, 911

Haemmerlé L., Woods T. E., Klessen R. S., Heger A., Whalen D. J., 2017, ArXiv e-prints:1705.09301

Hahn O., Abel T., 2011, MNRAS, 415, 2101

Haiman Z., 2006, New Astronomy Review, 50, 672

Hockney R. W., Eastwood J. W., 1988, Computer simulation using particles. Bristol: Hilger, 1988

Hosokawa T., Omukai K., Yorke H. W., 2013a, ApJ, 778, 178

Hosokawa T., Yorke H. W., Inayoshi K., Omukai K., Yoshida N., 2013b, ApJ, 778, 178

Hosokawa T., Hirano S., Kuiper R., Yorke H. W., Omukai K., Yoshida N., 2016, ApJ, 824, 119

Inayoshi K., Haiman Z., 2014, MNRAS, 445, 1549

Jeon M., Pawlik A. H., Bromm V., Milosavljević M., 2014, MNRAS, 440, 3778

Johnson J. L., Khochfar S., Greif T. H., Durier F., 2011, MNRAS, 410, 919

Kitsionas S., Whitworth A. P., 2002, MNRAS, 330, 129

Kormendy J., Ho L. C., 2013, ARA\&A, 51, 511

Krumholz M. R., McKee C. F., Klein R. I., 2004, ApJ, 611, 399

Latif M. A., Volonteri M., 2015, MNRAS, 452, 1026

Latif M. A., Schleicher D. R. G., Bovino S., Grassi T., Spaans M., 2014, ApJ, 792, 78

Latif M. A., Bovino S., Grassi T., Schleicher D. R. G., Spaans M., 2015, MNRAS, 446, 3163

Latif M. A., Schleicher D. R. G., Hartwig T., 2016, MNRAS, 458, 233

Loeb A., Rasio F. A., 1994, ApJ, 432, 52

Madau P., Rees M. J., 2001, ApJ, 551, L27

Milosavljević M., Couch S. M., Bromm V., 2009, ApJ, 696, L146

Mortlock D. J. et al., 2011, Nature, 474, 616

Planck Collaboration et al., 2014, A\&A, 571, A16

Regan J. A., Haehnelt M. G., 2009a, MNRAS, 396, 343

Regan J. A., Haehnelt M. G., 2009b, MNRAS, 393, 858

Regan J. A., Johansson P. H., Haehnelt M. G., 2014a, MNRAS, 439, 1160

Regan J. A., Johansson P. H., Wise J. H., 2014b, ApJ, 795, 137

Regan J. A., Johansson P. H., Wise J. H., 2015, MNRAS, 449, 3766

Regan J. A., Johansson P. H., Wise J. H., 2016, MNRAS

Regan J. A., Visbal E., Wise J. H., , Haiman Z., Johansson P. H., Bryan G. L., 2017, Nature Astronomy, 1, 0075 
Safranek-Shrader C., Agarwal M., Federrath C., Dubey A., Milosavljević M., Bromm V., 2012, MNRAS, 426, 1159

Safranek-Shrader C., Montgomery M. H., Milosavljević M., Bromm V., 2016, MNRAS, 455, 3288

Schauer A. T. P., Regan J., Glover S. C. O., Klessen R. S., 2017, MNRAS, 471, 4878

Schleicher D. R. G., Palla F., Ferrara A., Galli D., Latif M., 2013, A\&A, 558, A59

Shang C., Bryan G. L., Haiman Z., 2010, MNRAS, 402, 1249

Shu F. H., 1977, ApJ, 214, 488

Smith B. D. et al., 2017, MNRAS, 466, 2217

Stacy A., Bromm V., Lee A. T., 2016, MNRAS, 462, 1307

Sugimura K., Omukai K., Inoue A. K., 2014, MNRAS, 445, 544

Tanaka T., Haiman Z., 2009, ApJ, 696, 1798

Tanaka T. L., Li M., 2014, MNRAS, 439, 1092

Turk M. J., Smith B. D., Oishi J. S., Skory S., Skillman S. W., Abel T., Norman M. L., 2011, ApJS, 192, 9

Volonteri M., 2010, A\&A Rev., 18, 279

Wang P., Li Z. Y., Abel T., Nakamura F., 2010, ApJ, 709, 27

Whalen D., Abel T., Norman M. L., 2004, ApJ, 610, 14

Wise J. H., Turk M. J., Abel T., 2008, ApJ, 682, 745

Wolcott-Green J., Haiman Z., 2012, MNRAS, 425, L51

Wolcott-Green J., Haiman Z., Bryan G. L., 2017, MNRAS, 469, 3329

Woods T. E., Heger A., Whalen D. J., Haemmerlé L., Klessen R. S., 2017, ApJ, 842, L6

Wu X. B. et al., 2015, Nature, 518, 512

Yoshida N., Abel T., Hernquist L., Sugiyama N., 2003, ApJ, 592, 645

This paper has been typeset from a $\mathrm{T}_{\mathrm{E}} \mathrm{X} / \mathrm{LT}_{\mathrm{E}} \mathrm{X}$ file prepared by the author.

\section{APPENDIX A: SMARTSTAR TESTS}

As part of the integration of our Smartstar particle into Enzo we rigorously tested the sink particle technique used in this work. We tested the implementation using both the Singular Isothermal Sphere test (Shu 1977) and by analysing the results from a Rotating Cloud test (Boss \& Bodenheimer 1979; Burkert \& Bodenheimer 1993).

\section{A1 Collapse of a Singular Isothermal Sphere}

The collapse of a singular isothermal sphere with a $\rho(r) \propto r^{-2}$ density profile produces a constant flux of mass through spherical shells i.e. a constant accretion rate. We compute the collapse of a singular isothermal sphere to test whether our model of sink particle accretion reflects this collapse behaviour. We follow the work of Federrath et al. (2010) in conducting this numerical test. The sphere is set to have a truncation radius of $\mathrm{R}=5 \times 10^{16} \mathrm{~cm}$, a density at this radius of $\rho(\mathrm{R})=3.82 \times 10^{-18} \mathrm{~g} \mathrm{~cm}^{-3}$ and therefore a total mass of $3.02 \mathrm{M}_{\odot}$. The sphere is initialized at a temperature of $10 \mathrm{~K}$, the mean molecular weight of the gas is set to 3.0 resulting in a sound speed of $0.166 \mathrm{~km} \mathrm{~s}^{-1}$. These values lead to a large instability parameter for the sphere of $\mathrm{A}=29.3$ with $\mathrm{A}=4 \pi G \rho(R) R^{2} / c_{s}^{2}$ (Shu 1977). We ran the test at a maximum refinement level of 10 leading to a minimal grid spacing of $\Delta x \sim 3.05 \times 10^{-5} \mathrm{pc}$. No radiative cooling or chemical reactions are calculated during the collapse.

In the upper panel of Figure A1 we show the mass, in solar masses, as a function of time. The Smartstar particle mass increases monotonically as it accretes matter eventually accreting the entire envelope $\left(3.02 \mathrm{M}_{\odot}\right)$. The slope of the line is $1.5 \times 10^{-4}$ $\mathrm{M}_{\odot} / \mathrm{yr}$ in excellent agreement with the analytical solution predicted by Shu (1977). In the lower panel of Figure A1 we show the mass accretion rate during this time. The test predicts a constant accretion rate of $1.5 \times 10^{-4}$ and this is confirmed by our test. The bump at approximately 17000 yrs corresponds to the SmartStar particle accreting the last of the matter and at this point the accretion rate fluctuates mildly before falling to zero.

We also examine the behaviour of the gas itself and the mass that is accreted by the Smartstar particle. In Figure A2 we see the density profile, radial velocity profile and the mass accretion through spherical shells as a function of radius. Overlaid on each plot are the analytical expressions for the collapse taken from Shu (1977). The agreement in all three profiles is excellent with mild deviations only appearing once the the entire envelope has been accreted at $\mathrm{T} \sim 17000 \mathrm{yrs}$.

\section{A2 Rotating Cloud Core Fragmentation Test}

Finally, we also analyse the behaviour of the Smartstar particles when the gas is subject to rotation and collapse. This is the classic Boss \& Bodenheimer (1979) test. It is a standard test for examining fragmentation in hydrodynamical simulations (Burkert et al. 1997; Federrath et al. 2010). We employ a setup very similar to that of Burkert \& Bodenheimer (1993), Bate \& Burkert (1997) and Federrath et al. (2010). The cloud is initialised with the following parameters: radius $\mathrm{R}=5 \times 10^{16} \mathrm{~cm}$, constant density $\rho_{0}=3.82 \times 10^{-18} \mathrm{gcm}^{-3}$, mass $=1 \mathrm{M}_{\odot}$, angular velocity, $\Omega=7.2 \times 10^{-13} \mathrm{rads}^{-1}$ (ratio of rotational to gravitational energy $\beta=0.16$ ), sound speed $=0.166 \mathrm{~km} \mathrm{~s}^{-1}$ (ratio of thermal to gravitational energy $\alpha=0.26$ ), global free-fall time $\mathrm{t}_{\mathrm{ff}}=1.075 \times 10^{12} \mathrm{~s}=3.41 \times 10^{4} \mathrm{yr}$. The cloud is then subject to a density perturbation with an $\mathrm{m}=2$ mode: $\rho=\rho_{0}[1+$ $0.1 \operatorname{Cos}(2 \phi)]$, where $\phi$ is the azimuthal angle. We use an isothermal equation of state throughout the test.

The initial rotation $(\beta=0.16)$ forces the gas cloud to collapse to a disk with a central bar due to the $\mathrm{m}=2$ initial density perturbation. Smartstar particles then form at the ends of the bar once the conditions for particle formation are met. The test was run at a maximum refinement level of 5 with a minimum cell size of 16.32 AU $\left(2.44 \times 10^{14} \mathrm{~cm}\right)$. The evolution of the system is shown in Figure A3. The figures show the initial formation of the disk and bar structure face on. Two Smart Star particles form at either end of the bar at the same time due to the inherent symmetry of the system. A third fragment then forms at the centre of the cloud core. Subsequently the two end fragments move along the bar and merge with the central fragment. The results of this test are in excellent agreement with similar tests carried out by Federrath et al. (2010). 


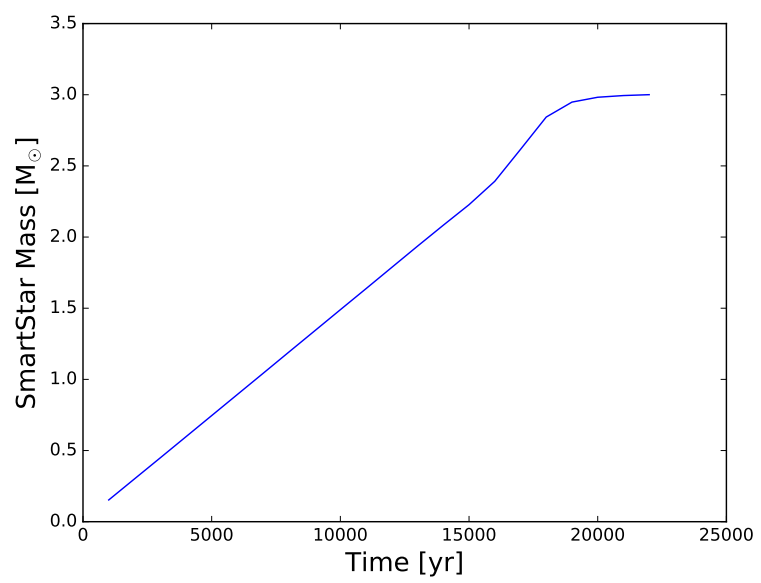

(a) The Mass of the SmartStar particle as a function of time. The slope of the line is approximately $1 \times 10^{-4} \mathrm{M}_{\odot} \mathrm{yr}^{-1}$ in excellent agreement with the analytical predictions (Shu 1977).

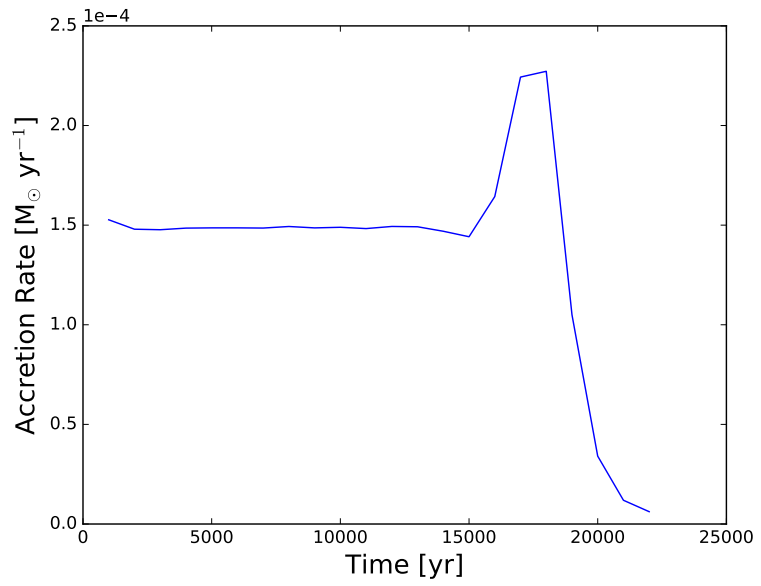

(b) The mass accretion history of the SmartStar particle over the timeframe of the isothermal collapse. The mass accretion rate is constant at $1 \times 10^{-4} \mathrm{M}_{\odot} \mathrm{yr}^{-1}$ until the envelope is nearly entirely accreted at $\mathrm{T}$ $\sim 17000 \mathrm{yrs}$.

Figure A1. The characteristics of the SmartStar particle undergoing isothermal collapse. In the top panel (a) we show the mass of the SmartStar particle as a function of time. In the bottom panel we show the mass accretion rate as a function of time. From both panels we see a constant mass accretion rate of $\dot{M}=1.5 \times 10^{-4} \mathrm{M}_{\odot} \mathrm{yr}^{-1}$.

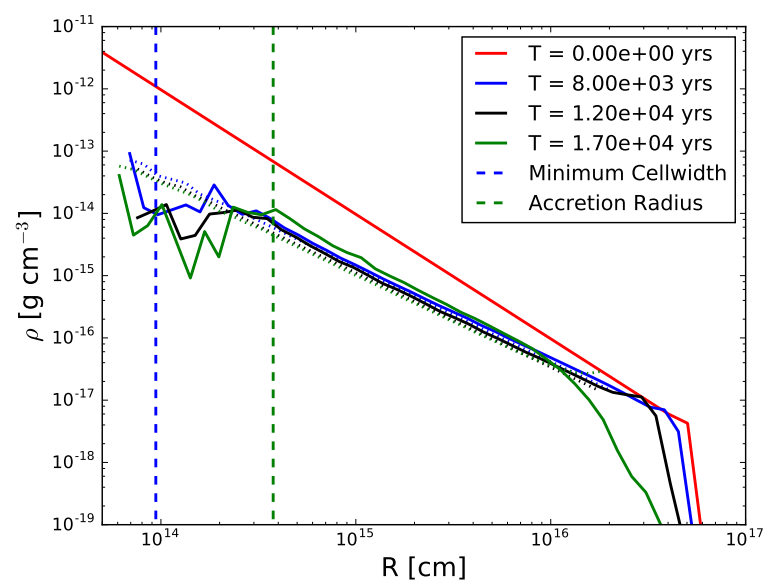

(a) The density profile of the gas as a function of radius for multiple output times. The density profile is initially $\rho(R) \propto r^{-2}$ and falls to $\rho(R) \propto r^{-1.5}$ as the collapse proceeds. The dotted lines are the analytical predictions, they fall almost exactly on top of the numerical results.

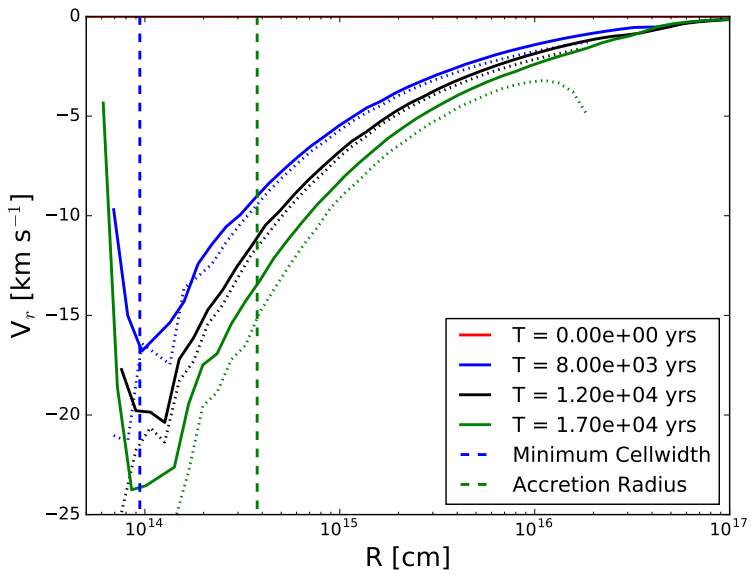

(b) The radial velocity of gas undergoing isothermal collapse. The gas is highly supersonic and again matches the analytical predictions (dotted lines) very closely.

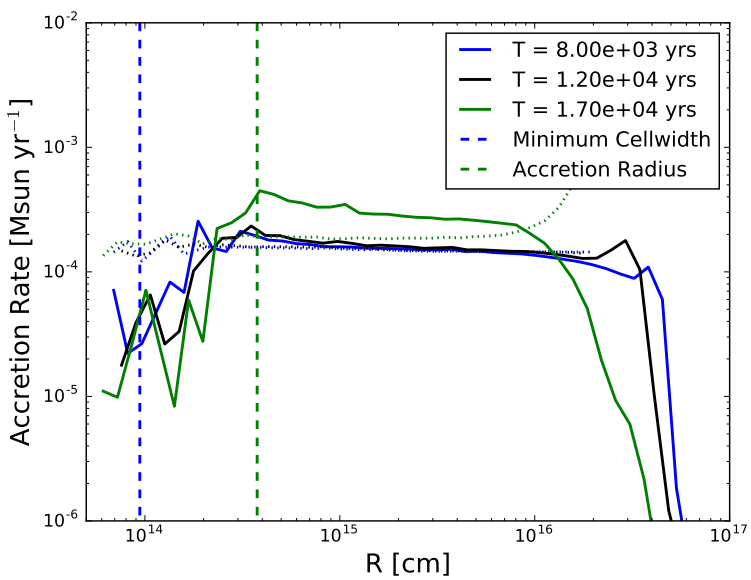

(c) The mass accretion history again showing the constant mass accretion over spherical shells of $\dot{M} \sim 1 \times 10^{-4} \mathrm{M}_{\odot} / \mathrm{yr}$.

Figure A2. Properties of the gas surrounding the accreting SmartStar particle. 


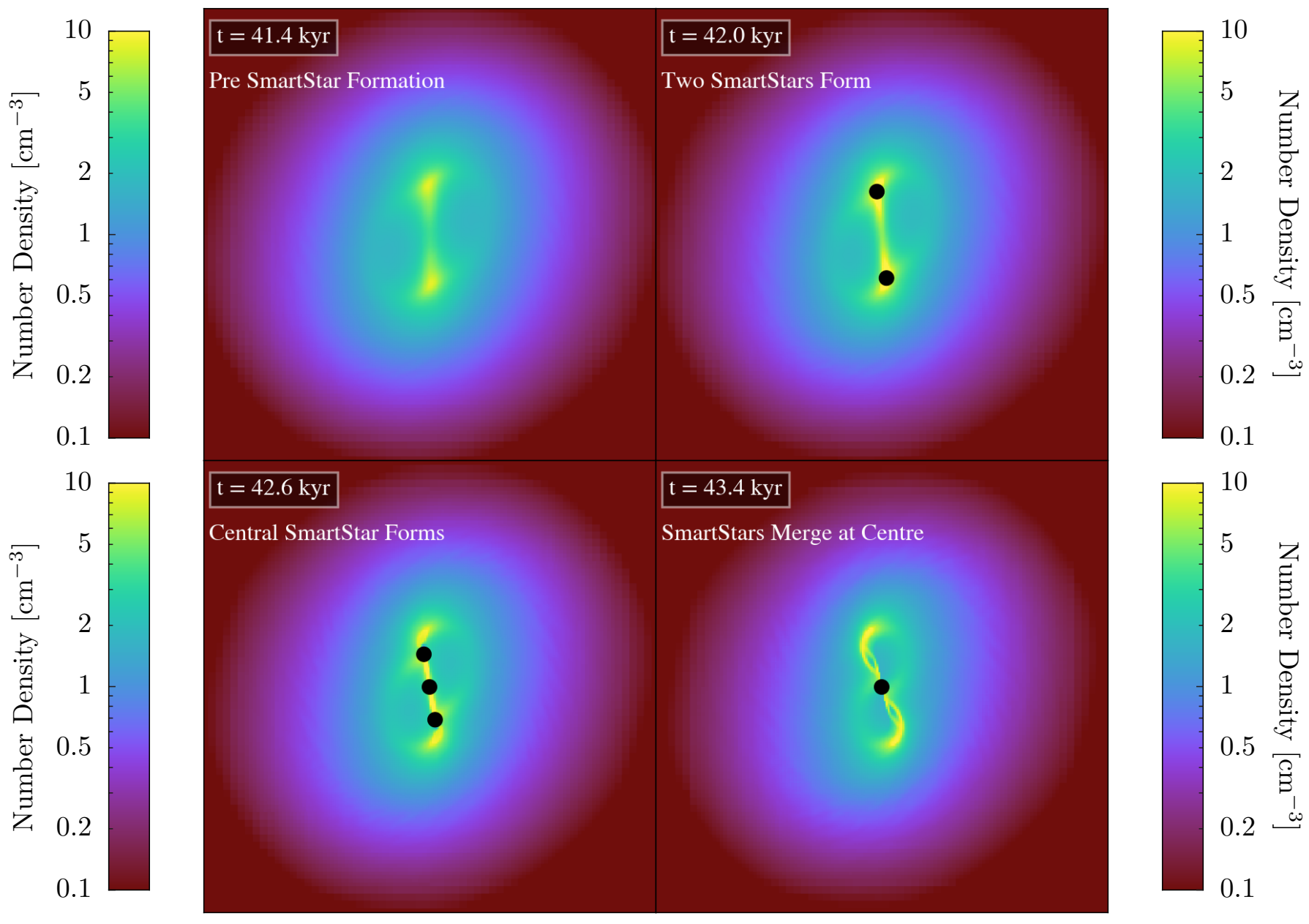

Figure A3. Projections of the rotating cloud test uses to examine the formation and merging properties of the Smart Star particles. In the top left panel at $\mathrm{t}=41.4 \mathrm{kyrs}$ the gas cloud has flattened into a disk and the threshold for SmartStar particle formation is about to be realised. In the top right panel SmartStar particles form at either end of a bar. As the simulation continues a third particle forms at the centre of the bar. By $t=43.4$ kyrs the three SmartStar particles have merged at the centre of the cloud. 